\title{
EDITORIAL \\ Achieving optimal outcomes with deep brain stimulation for posttraumatic stress disorder
}

\author{
Michael D. Staudt, MD, MSc, ${ }^{1}$ Ilknur Telkes, $\mathrm{PhD},{ }^{2}$ and Julie G. Pilitsis, MD, PhD ${ }^{1,2}$ \\ Departments of ${ }^{1}$ Neurosurgery and ${ }^{2}$ Neuroscience and Experimental Therapeutics, Albany Medical College, Albany, New York
}

$\mathrm{P}$ OSTTRAUMATIC stress disorder (PTSD) is a complex and devastating disease that can develop from experiencing or witnessing a traumatic event, often as a result of exposure to combat, physical or sexual violence, or life-threatening situations. PTSD is a markedly heterogeneous disorder, due to the severity of the initiating trauma, clinical manifestation of symptoms, and presence of other psychiatric comorbidities. ${ }^{1}$ In fact, according to the 5th edition of the Diagnostic and Statistical Manual of Mental Disorders, there are 636,120 symptom combinations that can lead to a PTSD diagnosis. ${ }^{2}$ There is considerable inconsistency regarding the identification of specific patient characteristics that can predict treatment outcomes following conventional therapy, with a significant proportion of patients with PTSD experiencing continued or residual symptoms after comprehensive cognitive behavioral therapy. ${ }^{3}$ This parallels treatment nonresponders in other psychiatric disorders; for example, up to $30 \%$ of patients with major depression do not respond to optimized multimodal therapy. ${ }^{4}$

In their paper titled "Neurostimulation for treatmentresistant posttraumatic stress disorder: an update on neurocircuitry and therapeutic targets," Larkin et al. provide a well-written and thoughtful review of the PTSD literature, including a discussion of the underlying neurobiology and current status of neuromodulation research and treatments. ${ }^{5}$ Because PTSD results from dysfunctional neural circuitry, a better understanding of such pathways is essential to develop neuromodulatory targets. Preclinical models remain an important tool for study; however, animal models of PTSD cannot accurately translate a patient's experience involving such complex behavioral and cognitive processing. Therefore, innovations in neuroimaging have advanced our knowledge of the anatomical and functional pathophysiology of PTSD. Larkin et al. have crafted a succinct analysis of relevant preclinical and clinical studies, ultimately providing a perspective on the current state of neuromodulation that is relevant to neurosurgeons.
The use of deep brain stimulation (DBS) has been the focus of trials in a number of psychiatric conditions including depression, obsessive-compulsive disorder (OCD), and Tourette syndrome, with promising but sometimes inconsistent results. Although DBS has the potential to modulate widespread networks, the challenges encountered regarding its use in psychiatric disorders may be attributable to the nonselective stimulation of multiple structures. Currently, there are 2 ongoing clinical trials of DBS for PTSD: an open-label study of 5 subjects targeting the subgenual cingulate (NCT03416894), and a randomized parallel study of 6 subjects targeting the basolateral amygdala (BLA) (NCT02091843). The subgenual cingulate has classically been targeted in DBS for depression; however, both anatomical and functional studies have demonstrated structural abnormalities in patients with PTSD. ${ }^{6,7}$ Indeed, there is complex and rich connectivity of the multiple brain regions involved in fear and emotional arousal, and patients with PTSD have demonstrated concurrent activation of the cingulate cortex and amygdala in imaging studies during exposure to stimuli. ${ }^{8}$

There has yet to be any published data regarding these 2 ongoing clinical trials, and study completion is not expected for a few years. However, the ongoing results of 2 patients undergoing bilateral BLA DBS have been reported in a number of publications and abstracts. The first subject with PTSD to undergo DBS was a 48-yearold male combat veteran; at 8 months, he experienced a $37.8 \%$ improvement in his Clinically-Administered PTSD Scale (CAPS) score. ${ }^{9}$ When this improvement peaked at approximately $15-18$ months, the patient began to decompensate, as characterized by depression and suicidal gestures, which was not attributed to an exacerbation of his PTSD or changes in DBS stimulation settings..$^{10}$ The second patient, a 38-year-old male combat veteran, demonstrated improved engagement in social activities and anger regulation at his 6-month follow-up. ${ }^{11}$ Interestingly, both subjects experienced varied emotions when differ- 
ent contacts were acutely tested at different stimulation strengths, suggesting a unique avenue for studying emotional valence outside PTSD. ${ }^{12}$

Psychiatric disorders are complex disease states that are influenced by cognitive, behavioral, and emotional factors, and their underlying pathophysiology is probably attributable to a dysfunction of networks..$^{13}$ For example, the brain regions associated with PTSD include the amygdala, hippocampal formation, and prefrontal cortex. The amygdala, and in particular the BLA, is responsible for the integration of internal and external stimuli, and has extensive cortical and subcortical connections. ${ }^{14}$ Our understanding of the structural connectivity underlying psychiatric disease has been improved with recent advances in imaging technology, such as delineating white matter connections implicated in affective processing. ${ }^{15}$ Previously, Riva-Posse et al. identified the fact that patients with treatment-resistant depression who responded to DBS were being stimulated along similar fiber pathways. ${ }^{16}$ The authors' subsequent prospective study identified the optimal DBS contacts for chronic stimulation by matching preoperative deterministic with postoperative probabilistic tractography. ${ }^{17}$ As such, targeting based on patientspecific data will probably be essential for DBS planning in psychiatric disorders, including PTSD.

There are lessons to be learned from the literature on DBS for other psychiatric disorders; for example, early open-label trials for treatment-resistant depression and bipolar depression were promising, ${ }^{18}$ although the randomized clinical trials that followed failed to demonstrate significant improvement. ${ }^{19,20}$ The initial reaction was to refine electrode placement in an attempt to identify the "best target"; however, it has become increasingly recognized that a single target does not exist, and identifying diseasespecific and patient-specific commonalities is likely to be necessary. For example, ambiguity has been identified regarding the labeling of DBS targets for OCD, such as separately classified targets having similar anatomical coordinates. ${ }^{21}$ Due to the close proximity of subcortical structures and white matter tracts, it is likely that DBS results in multiple targets being stimulated, and conversely that an efficacious target may be stimulated by multiple electrode trajectories. As such, proposing a single, ideal target for PTSD may be impractical, and this is further confounded by the considerable heterogeneity introduced by variations in PTSD diagnosis and clinical assessment.

The limitations of current preclinical and clinical studies and the technical limitations of imaging modalities necessitate a multimodality approach to improve the sensitivity and specificity of objective measures. To this end, functional and structural imaging data can be fused with neuronal oscillatory dynamics, such as time-locked evoked or event-related potentials or magnetic fields, obtained through electroencephalography (EEG) and magnetoencephalography modalities. ${ }^{22}$ The research on EEG signals in PTSD has commonly shown a hemispheric asymmetry in the parietal region in alpha band power associated with arousal and attentional disruption. ${ }^{23}$ The low temporal resolution of functional MRI can be improved by a simultaneous EEG recording, or information from each modality can be combined through multivariate analysis.
Furthermore, despite the limited number of studies, EEG has been used in neurofeedback studies in patients with PTSD wherein patients attempt to modulate their brain signals in a real-time EEG setup through training. ${ }^{24}$

In this scope, DBS surgery provides an unparalleled opportunity to record neural signals from deep brain structures via micro- or macroelectrodes and cortical structures via electrocorticography arrays or strip electrodes. Real-time monitoring of field potentials and unit activities can inform DBS lead placement, elucidate disease electrophysiological components, evaluate immediate neural response to electrical stimulation therapy, and lead to the development of novel hardware designs more suited to selected targets and closed-loop systems in patients with PTSD. ${ }^{25}$

Advancing the utility of DBS for PTSD will require a rigorous approach. Reporting of the stimulation target must be made clear, including an accurate reporting of stereotactic coordinates and active contacts. Outcome measures should be robust; in addition to assessing CAPS scores, it is important to include validated metrics of depression, anxiety, and disability, because these can inform treatment outcomes outside of disease-specific metrics. Future emerging technologies enabling simultaneous stimulation and sensing from DBS electrodes and storage of the recorded data for further analysis will advance our understating of PTSD pathophysiology and aid in the development of biomarker-guided neuromodulation strategies.

https://thejns.org/doi/abs/10.3171/2020.5.JNS201127

\section{References}

1. DiMauro J, Carter S, Folk JB, Kashdan TB. A historical review of trauma-related diagnoses to reconsider the heterogeneity of PTSD. J Anxiety Disord. 2014;28(8):774-786.

2. Galatzer-Levy IR, Bryant RA. 636,120 ways to have posttraumatic stress disorder. Perspect Psychol Sci. 2013;8(6): 651-662.

3. Cloitre M. The "one size fits all" approach to trauma treatment: should we be satisfied? Eur J Psychotraumatol. 2015; 6:27344.

4. Rush AJ, Trivedi MH, Wisniewski SR, et al. Acute and longer-term outcomes in depressed outpatients requiring one or several treatment steps: a STAR*D report. Am J Psychiatry. 2006;163(11):1905-1917.

5. Larkin MB, McGinnis JP, Snyder RI, et al. Neurostimulation for treatment-resistant posttraumatic stress disorder: an update on neurocircuitry and therapeutic targets. J Neurosurg. Published online July 31, 2020. doi:10.3171/2020.4.JNS2061

6. Herringa R, Phillips M, Almeida J, et al. Post-traumatic stress symptoms correlate with smaller subgenual cingulate, caudate, and insula volumes in unmedicated combat veterans. Psychiatry Res. 2012;203(2-3):139-145.

7. Tuescher O, Protopopescu X, Pan H, et al. Differential activity of subgenual cingulate and brainstem in panic disorder and PTSD. J Anxiety Disord. 2011;25(2):251-257.

8. Fredrikson M, Faria V. Neuroimaging in anxiety disorders. Mod Trends Pharmacopsychiatry. 2013;29:47-66.

9. Langevin JP, Koek RJ, Schwartz HN, et al. Deep brain stimulation of the basolateral amygdala for treatment-refractory posttraumatic stress disorder. Biol Psychiatry. 2016;79(10): e82-e84.

10. Langevin JP, Koek RJ, Schwartz HN, et al. Neuromodulation 
for treatment-refractory PTSD. Fed Pract. 2017;34(2)(suppl 2):20S-33S.

11. Koek R, Langevin J, Krahl S, et al. Basolateral amygdala deep brain stimulation for treatment refractory combat PTSD: data from the first two cases. Paper presented at: 3rd International Brain Stimulation Conference; February 24-27, 2019; Vancouver, BC, Canada.

12. Lai G, Langevin JP, Koek RJ, et al. Acute effects and the dreamy state evoked by deep brain electrical stimulation of the amygdala: associations of the amygdala in human dreaming, consciousness, emotions, and creativity. Front Hum Neurosci. 2020;14:61.

13. Staudt MD, Herring EZ, Gao K, et al. Evolution in the treatment of psychiatric disorders: from psychosurgery to psychopharmacology to neuromodulation. Front Neurosci. 2019;13: 108.

14. Garrido MI, Barnes GR, Sahani M, Dolan RJ. Functional evidence for a dual route to amygdala. Curr Biol. 2012;22(2): 129-134.

15. Sweet JA, Thyagaraj S, Chen Z, et al. Connectivity-based identification of a potential neurosurgical target for mood disorders. J Psychiatr Res. 2020;125:113-120.

16. Riva-Posse P, Choi KS, Holtzheimer PE, et al. Defining critical white matter pathways mediating successful subcallosal cingulate deep brain stimulation for treatment-resistant depression. Biol Psychiatry. 2014;76(12):963-969.

17. Riva-Posse P, Choi KS, Holtzheimer PE, et al. A connectomic approach for subcallosal cingulate deep brain stimulation surgery: prospective targeting in treatment-resistant depression. Mol Psychiatry. 2018;23(4):843-849.

18. Holtzheimer PE, Kelley ME, Gross RE, et al. Subcallosal cingulate deep brain stimulation for treatment-resistant unipolar and bipolar depression. Arch Gen Psychiatry. 2012; 69(2):150-158

19. Holtzheimer PE, Husain MM, Lisanby SH, et al. Subcallosal cingulate deep brain stimulation for treatment-resistant depression: a multisite, randomised, sham-controlled trial. Lancet Psychiatry. 2017;4(11):839-849.

20. Dougherty DD, Rezai AR, Carpenter LL, et al. A randomized sham-controlled trial of deep brain stimulation of the ventral capsule/ventral striatum for chronic treatment-resistant depression. Biol Psychiatry. 2015;78(4):240-248.

21. Raviv N, Staudt MD, Rock AK, et al. A systematic review of deep brain stimulation targets for obsessive compulsive disorder. Neurosurgery. Published online July 2, 2020. doi: 10.1093/neuros/nyaa249

22. Zotev V, Phillips R, Misaki M, et al. Real-time fMRI neurofeedback training of the amygdala activity with simultaneous EEG in veterans with combat-related PTSD. Neuroimage Clin. 2018;19:106-121.

23. Metzger LJ, Paige SR, Carson MA, et al. PTSD arousal and depression symptoms associated with increased right-sided parietal EEG asymmetry. J Abnorm Psychol. 2004;113(2): 324-329.

24. Kluetsch RC, Ros T, Théberge J, et al. Plastic modulation of PTSD resting-state networks and subjective wellbeing by EEG neurofeedback. Acta Psychiatr Scand. 2014;130(2): 123-136.

25. Deeb W, Giordano JJ, Rossi PJ, et al. Proceedings of the Fourth Annual Deep Brain Stimulation Think Tank: a review of emerging issues and technologies. Front Integr Neurosci. 2016;10:38

\section{Disclosures}

Dr. Pilitsis is a consultant for Boston Scientific, Nevro, TerSera, Medtronic, and Abbott, and receives grant support from Medtronic, Boston Scientific, Abbott, Nevro, TerSera, and NIH (2R01CA166379-06 and NIH U44NS115111). She is a medical advisor and a speaker's bureau member for Aim Medical Robotics and Karuna, and has stock equity in both companies.

\section{Correspondence}

Julie G. Pilitsis: jpilitsis@yahoo.com.

\section{INCLUDE WHEN CITING}

Published online July 31, 2020; DOI: 10.3171/2020.5.JNS201127.

\section{Response}

\section{Benjamin Larkin, MD, PharmD, Ashwin Viswanathan, MD, and Sameer A. Sheth, MD, PhD}

Department of Neurosurgery, Baylor College of Medicine, Houston, Texas

We thank Drs. Staudt, Telkes, and Pilitsis for their thoughtful commentary accompanying our article. The context they provide on the current state of the art in psychiatric neurosurgery serves as a very helpful framework within which to place our specific discussion regarding PTSD.

There is one particular theme from their commentary that we would like to emphasize and expand upon: that of treatment personalization. We agree that surgical neuromodulation for severe PTSD must avail itself of lessons from our field's experience with other neurological and psychiatric disorders. DBS for OCD, depression, and Tourette syndrome, for example, has been delivered across several target structures in the attempt to find the "optimal" target. When planning DBS for Parkinson's disease (PD), however, there is no single target that optimally benefits all patients. We consider the patient's symptoms (e.g., tremor, bradykinesia, rigidity, and gait difficulty); goals (e.g., medication reduction); and comorbidities (e.g., advanced age and cognitive impairment) - all of which factor into the target choice for that patient. Thus, we have developed the concept that there is not a single optimal target across patients, but there may indeed be an optimal target for an individual patient. Just as we have this more nuanced approach to DBS for PD, we must develop an individual-specific approach to DBS for newer indications like PTSD.

For this reason, an important rate-limiting step is a more comprehensive understanding of the disorder itself, and of how different brain circuits contribute to different facets of the overall symptomatology. As we mentioned in our source article, an approach like the Research Domain Criteria (RDoC) project may help "orthogonalize" the constellations of symptoms onto axes with more neurobiological labels (e.g., systems related to fear, anxiety, reward valuation, attention, etc.), thereby facilitating consistency and contextualization of research in this patient population in comparison with others. As we start building a more thorough understanding of the disorder in general, we can then better appreciate the relative contributions of these dysfunctional systems at the individual level and truly personalize treatment decisions such as DBS target choice.

As an example of this type of approach in practice, we refer to an ongoing clinical trial (NCT03437928) we are running in which we are using DBS for depression. 
This trial, funded by the NIH BRAIN Initiative (UH3 NS103549), focuses on identifying individual-specific aspects of dysfunction in depression-relevant networks and using this information to personally tailor DBS therapy. We borrow from the world of epilepsy surgery the approach of intracranial recording and stimulation to derive a detailed understanding of the subject's brain networks regulating mood, affect, and cognition, as well as these networks' response to stimulation. We use this information to optimize the delivery of DBS therapy by narrowing the vast parameter space of stimulation to the subset of parameters that best restores the system to a "healthier" state. An approach such as this could of course be used to optimize and personalize DBS for any neurological or psychiatric disorder for which a network understanding is incomplete.

In our opinion, the future of functional neurosurgery and surgical neuromodulation will benefit from these ideas of understanding networks and individualizing therapy. Of paramount importance is the simultaneous application of a rigorous neuroethical framework to constrain our efforts and maintain the highest patient-centric standards. These principles must guide the growth of this field as we continue developing treatments for patients with severe refractory brain disorders.

\section{INCLUDE WHEN CITING}

Published online July 31, 2020; DOI: 10.3171/2020.5.JNS201672. 